\title{
MicroRNA-200b inhibits epithelial-mesenchymal transition and migration of cervical cancer cells by directly targeting RhoE
}

\author{
YAN-XIANG CHENG ${ }^{1}$, GAN-TAO CHEN ${ }^{2}$, CHUANG CHEN ${ }^{3}$, \\ QI-FAN ZHANG ${ }^{1}$, FENG PAN $^{4}, \mathrm{MIN} \mathrm{HU}^{1}$ and BING-SHU LI ${ }^{1}$ \\ ${ }^{1}$ Department of Obstetrics and Gynecology, Renmin Hospital of Wuhan University, Wuhan, \\ Hubei 430060; ${ }^{2}$ Department of Oncology, Third People's Hospital of Xiantao, Xiantao, Hubei 433000; \\ Departments of ${ }^{3}$ Breast and Thyroid Surgery and ${ }^{4}$ Orthopaedics, Renmin Hospital of Wuhan University, \\ Wuhan, Hubei 430060, P.R. China
}

Received February 2, 2015; Accepted January 4, 2016

DOI: $10.3892 / \mathrm{mmr} .2016 .4933$

\begin{abstract}
Previous studies have identified microRNA-200b (miR-200b) as a powerful regulator of epithelial-mesenchymal transition (EMT) via the control of gene expression. EMT is a critical event that is associated with the initiation of malignant tumor metastasis. A lack of E-cadherin expression and overexpression of vimentin are hallmarks of EMT. It is well-known that $\mathrm{RhoE}$, which is associated with regulation of the actin cytoskeleton and migration via alterations in cell motility, regulates the expression of E-cadherin, matrix metalloproteinase-9 (MMP-9) and vimentin. However, it remains to be elucidated whether miR-200b may alter the molecular behavior of RhoE. The present study aimed to determine whether miR-200b was able to regulate the EMT of cervical cancer, in order to control metastasis. In addition, the correlation between miR-200b and RhoE, E-cadherin and vimentin expression was investigated. Notably, miR-200b was shown to inhibit the function of RhoE and suppress the EMT of cervical cancer. Furthermore, HeLa cells were transfected with miR-200b mimics or inhibitors, and the protein expression levels of E-cadherin, MMP-9, vimentin and RhoE were subsequently detected. A Transwell assay was also conducted, in order to observe the metastatic ability of the HeLa cells. In addition, a luciferase reporter assay was performed using luciferase reporter vectors containing the full length 3'-untranslated region (UTR) of RhoE; miR-200b was able to significantly suppress relative luciferase activity by targeting the 3'-UTR of RhoE. These results suggested that miR-200b may markedly inhibit metastatic potential by regulating cell EMT and inhibiting RhoE; therefore, miR-200b
\end{abstract}

Correspondence to: Dr Chuang Chen, Department of Breast and Thyroid Surgery, Renmin Hospital of Wuhan University, 99 Zhang Zhidong Road, Wuhan, Hubei 430060, P.R. China E-mail: drccwhu@163.com

Key words: epithelial-mesenchymal transition, microRNA-200b, RhoE, cervical cancer may be considered an effective target for the treatment of patients with highly metastatic cervical cancer.

\section{Introduction}

Metastasis is the most common cause of cervical cancer-associated mortality. The American Cancer Society estimated that in 2015, 12,900 novel cases and 4,100 fatalities will result from cervical cancer in the United States (1). Screening and prevention programs have contributed to declining mortality rates, and surgical resection is available for the treatment of early stage tumors; however, the current prognosis for patients with cervical cancer remains poor, due to high rates of tumor metastasis (2-4). Improved understanding regarding the precise molecular mechanisms underlying metastasis remains a challenge in cancer research, in addition to identifying key targets against which therapeutic strategies may be directed.

Tumor metastasis is a complex, multistep process that includes dysregulation of tumor cell adhesion, cytoskeletal remodeling, and invasion of the surrounding organs or tissues. An essential factor of this morphogenetic transformation is referred to as epithelial-mesenchymal transition (EMT). During EMT, epithelial cells actively lose their cell-cell adhesive capacity and polarity, and acquire highly motile mesenchymal characteristics, as well as reduced intracellular interactions, which are essential for metastasis $(5,6)$. Notably, EMT is considered a crucial event that contributes to cancer progression, due to its ability to facilitate invasion and metastasis. Numerous studies have reported the importance of EMT, particularly with regards to the cell junction molecule E-cadherin, which can be functionally overexpressed in order to establish stable intracellular adhesion; therefore, loss of E-cadherin expression is considered a hallmark of EMT. Loss of E-cadherin expression and gain of vimentin expression (another marker of EMT) are common alterations that occur during human cancer invasion and metastasis $(7,8)$.

Various molecular processes are associated with the initiation and progression of EMT, including alterations in the expression of specific microRNAs (miRNAs). miRNAs are an abundant class of endogenous, small, non-coding regulatory RNA molecules, which are 17-25 nucleotides long. It is widely 
accepted that miRNAs regulate gene expression by recognizing imperfect complementary sites in the 3'-untranslated regions (3'-UTR) of target mRNA, which may lead to suppression of the target gene expression, or mRNA degradation (9). Previous studies have demonstrated that miRNAs have roles in almost all aspects of cancer biology, including invasion and metastasis (10-12). The human miRNA (miR)-200 family comprises five members: miR-200a, miR-200b, miR-200c, miR-429 and miR-141, which are able to regulate EMT by targeting the E-box-binding transcription factors, zinc E-box-binding homeobox 1 (ZEB1) and Smad-interacting protein 1 (SIP1/ZEB2) (13-15). In addition, ZEB1 and ZEB2 are essential suppressors of E-cadherin transcription and have been implicated in EMT. Previous studies have identified miR-200b as a powerful regulator of EMT, which is associated with cancer metastasis, via regulating the expression of genes, including E-cadherin and vimentin (14-16). Therefore, miR-200b is considered an important factor in the metastatic spread of cancer cells. It has been widely reported that the expression of miR-200b can influence invasion and metastasis of various types of cancer, including prostate (16), colon (17), hepatic (18), gastric (19) and bladder (20) cancer. In addition, miR-200b has been reported to be significantly differentially expressed between primary tumors and corresponding distant metastases in breast cancer (14); however, the association between miR-200b and metastatic processes in cervical cancer remain to be elucidated. The present study aimed to elucidate the potential molecular mechanism underlying the role of miR-200b in cervical cancer metastasis.

\section{Materials and methods}

Cell culture. The HeLa human cervical cancer cell line was obtained from the American Type Culture Collection (Manassas, VA, USA). The cells were cultured in Dulbecco's modified Eagle's medium (DMEM; Gibco; Thermo Fisher Scientific, Inc., Waltham, MA, USA), supplemented with $10 \%$ fetal bovine serum (FBS), $100 \mathrm{U} / \mathrm{ml}$ penicillin and $100 \mu \mathrm{g} / \mathrm{ml}$ streptomycin (all from Gibco; Thermo Fisher Scientific, Inc.) at $37^{\circ} \mathrm{C}$ in a humidified atmosphere containing $5 \% \mathrm{CO}_{2}$.

Cell transfection. Small interfering (si)RNA-RhoE, miRNA-200b mimics and inhibitors, and the negative control for siRNA-NC, were designed by and purchased from Guangzhou RiBoBio Co., Ltd. (Guangzhou, China). Cells were seeded in 6-well plates at a density of $40 \%$, and after an overnight incubation were transfected with the miRNAs, siRNAs, miRNA inhibitors and NC using Lipofectamine 2000 (Invitrogen; Thermo Fisher Scientific, Inc.) according to the manufacturer's protocol. After $6 \mathrm{~h}$, the culture medium was replaced with fresh DMEM. Total RNA and protein were subsequently extracted after $48 \mathrm{~h}$, for analysis using reverse transcription-quantitative polymerase chain reaction (RT-qPCR) or western blotting, respectively.

Cell migration assay. Transwell insert chambers containing an $8-\mu \mathrm{m}$ porous membrane (Corning Inc., Corning, NY, USA) were used to performed the migration assay. A total of $1 \times 10^{5}$ cells suspended in serum-free medium were placed on each upper chamber, and $500 \mu 1$ medium containing $10 \%$
FBS was added to the bottom chamber. The cells were incubated for $24 \mathrm{~h}$ at $37^{\circ} \mathrm{C}$ in a humidified incubator containing $5 \% \mathrm{CO}_{2}$. To quantify the number of migrated cells, cells on the upper surface of the Transwell membrane were removed using a cotton swab. The migrated cells on the reverse of the membrane were fixed in methanol, stained with crystal violet (Sigma-Aldrich, St. Louis, MO, USA) and images were captured under a microscope (BX31, Olympus Corporation, Tokyo, Japan) at x100 magnification. Six random fields from the triplicate membranes of each experimental group were observed.

Cell lysis and western blotting. Whole proteins were extracted from the cell lysates using radioimmunoprecipitation assay buffer (Beyotime Institute of Biotechnology, Hangzhou, China). The protein concentration was determined using the BCA Protein Assay kit (Thermo Fisher Scientific, Inc.). Equal quantity of proteins (25 $\mu \mathrm{g} /$ lane) were separated by $10-12 \%$ sodium dodecyl sulfate-polyacrylamide gel electrophoresis (Goodbio Technology, Wuhan, China) and were transferred onto polyvinylidene difluoride membranes (EMD Millipore, Billerica, MA, USA). The membranes were then blocked with $5 \%$ non-fat dried milk at room temperature and were incubated with monoclonal mouse anti-human $\operatorname{RhoE~}(1: 2,000$; cat. no. MAB6618; R\&D Systems, Minneapolis, MN, USA), monoclonal mouse anti-human E-cadherin $(1: 1,000$; cat. no. 3195), monoclonal rabbit anti-human vimentin (1:1,000; cat. no. 5741), polyclonal rabbit anti-human matrix metalloproteinase (MMP)-9 (1:1,000; cat. no. 4022) and monoclonal rabbit anti-human GAPDH (1:1,000; cat. no. 2118) antibodies (Cell Signaling Technology, Inc., Danvers, MA, USA). After being washed three times with Tris-buffered saline containing Tween $(0.02 \%)$, the membranes were incubated with goat anti-mouse $\operatorname{IgG} 2 b(1: 8,000$; cat. no. sc-2062) and goat anti-rabbit IgG2b (1:8,000; cat. no. sc-2004) horseradish peroxidase-conjugated secondary antibodies (Santa Cruz Biotechnology, Inc., Dallas, TX, USA) for $60 \mathrm{~min}$ at room temperature. The blots were visualized using an enhanced chemiluminescence system (EMD Millipore). GAPDH was used as an endogenous internal control. The immune complexes were quantified using Image J (version 1.46; National Institutes of Health, Bethesda, MA, USA)

$R N A$ preparation and $R T-q P C R$. Total RNA, including mRNA, was isolated from the cells post-transfection using TRIzol reagent (Invitrogen; Thermo Fisher Scientific, Inc.), according to the manufacturer's protocol and treated with $2 \mu \mathrm{l}$ RQ1 RNase-Free DNase (Promega Corporation, Madison, WI, USA). For mRNA analysis, cDNA was amplified from $2.0 \mu \mathrm{g}$ total RNA in a final volume of $20 \mu \mathrm{l}$ using the Revert Aid First Strand cDNA Synthesis kit (Thermo Fisher Scientific, Inc.). The primers used for the RT reaction were miR200b, 5'-GTCGTATCCAGTGCAGGGTCCGAGGTATTCGCACT GGATACGACTCATCAT-3' and U6, 5'-CGCTTCACGAAT TTGCGTGTCA-3'. The reaction was incubated at $42^{\circ} \mathrm{C}$ for $15 \mathrm{~min}$, then heated to $95^{\circ} \mathrm{C}$ for $5 \mathrm{~min}$ and finally incubated at $5^{\circ} \mathrm{C}$ for $5 \mathrm{~min}$. Human GAPDH was used as an internal control. The PCR primer sequences were as follows: RhoE, F 5'-ATA GAGTTGAGCCTGTGGGACAC-3' and R 5'-AGGGTCTCT GGTCTACTGATGTC-3'; and GAPDH, F 5'-TGCACCACCA 
ACTGCTTAGC-3' and R 5'-GGCATGGACTGTGGTCAT GAG-3', miR-200b, F 5'-GCGGCTAATACTGCCTGGTAA-3' and R 5'-GTGCAGGGTCCGAGGT-3', U6, F 5'-CGCTTC GGCAGCACATATACTA-3' and R 5'-CGCTTCACGAAT TTGCGTGTCA-3' obtained from Shanghai GenePharma Co., Ltd. (Shanghai, China). The RT-qPCR reaction was performed using SYBR Green Master mix (Applied Biosystems; Thermo Fisher Scientific, Inc.), with the following cycling conditions: $95^{\circ} \mathrm{C}$ for $10 \mathrm{~min}$, followed by 40 cycles at $95^{\circ} \mathrm{C}$ for $15 \mathrm{sec}$ and $60^{\circ} \mathrm{C}$ for $1 \mathrm{~min}$. RT-qPCR was performed using a StepOnePlus system (Applied Biosystems; Thermo Fisher Scientific, Inc.). For quantification of miR-200b, similar to the mRNA RT-qPCR, miRNA expression levels were analyzed using specific primers with U6 as an internal control. The primers were purchased from Guangzhou RiBoBio Co., Ltd. The results were analyzed using the $2^{-\Delta \Delta \mathrm{Cq}}$ method relative to U6 or GAPDH expression (21).

Luciferase assay. The 3'-UTR of RhoE was amplified by PCR using human genomic DNA and cloned into the XhoI site downstream of luciferase in the pLUC vector (Promega Corporation). A pLUC construct containing a mutated 3'-UTR of RhoE that lacked the seed sequence of miR-200b was also synthesized. For the luciferase assay, HEK293T cells (American Type Culture Collection) were seeded into 24-well plates and transfected with $200 \mathrm{ng}$ pLMP or pLMP-has-miR-200b vector (Guangzhou RiBoBio Co., Ltd.) alongside $20 \mathrm{ng}$ wild type or mutant RhoE 3'-UTR using Lipofectamine 2000 (Thermo Fisher Scientific, Inc.) at room temperature. Cells were collected after $48 \mathrm{~h}$ and were analyzed using the Dual-Luciferase Reporter Assay system (Promega Corporation). All experiments were performed four times and normalized to Renilla luciferase activity.

Statistical analysis. All experiments were performed independently in triplicate. Student's t-test (two-tailed) and one-way analysis of variance were performed in order to analyze the data. SPSS 19.0 software (IBM SPSS, Armonk, NY, USA) was used to analyze the data. Data are expressed as mean \pm standard error. $\mathrm{P}<0.05$ was considered to indicate a statistically significant difference.

\section{Results}

miR-200b expression regulates the EMT of HeLa cells. Loss of E-cadherin expression and increased invasiveness are markers of EMT. To determine whether miR-200b was able to regulate the EMT of cervical cancer cells, the expression levels of E-cadherin and vimentin were detected in HeLa cells transfected with miR-200b mimics, inhibitors, or a negative control. The expression levels of miR-200b were analyzed by RT-qPCR with U6 RNA as an internal control. As presented in Fig. 1A, the relative expression levels of miR-200b were 14-fold higher in the cells transfected with the miR-200b mimic, as compared with in the negative control cells (mimic-NC). In addition, the cells transfected with the miR-200b inhibitor exhibited a $~ 60 \%$ downregulation in miR-200b expression.

Overexpression of miR-200b in the HeLa cells resulted in upregulated E-cadherin and downregulated vimentin protein expression levels, as compared with in the negative control, as determined by western blotting (Fig. 1B-D). Furthermore, post-transfection with the miR-200b inhibitor E-cadherin expression was reduced and vimentin expression was increased (Fig. 1B-D). The expression levels of MMP-9 were also markedly decreased in the HeLa cells transfected with miR-200b mimics. Conversely, transfection with the miR-200b inhibitor significantly suppressed MMP-9 protein expression (Fig. 1B and E). These results suggest that metastasis of HeLa cells may, at least partially, be attributed to MMP-9 upregulation. In addition, miR-200b may be considered a powerful regulator of EMT in HeLa cells, due to the regulation of E-cadherin and vimentin expression. Loss of miR-200b expression may therefore have the potential to promote cervical cancer cell migration by initiating EMT.

miR-200b levels regulate HeLa cell migration. The present study investigated the effects of miR-200b on the migration of HeLa cells using a Transwell migration assay. Transfection of HeLa cells with miR-200b mimics suppressed cell migratory ability. Conversely, transfection of the cells with a miR-200b inhibitor resulted in a marked decrease in migratory ability (Fig. 1F). These results indicate that miR-200b levels may regulate HeLa cell migration.

$m i R-200 b$ directly targets $R h o E$. To explore the mechanisms underlying miR-200b-mediated reductions in cell metastasis, potential miR-200b target genes were investigated using online miRNA target prediction databases (Targetscan, targetscan.org; microrna.org/microrna/home.do and genie.weizmann.ac.il/pubs/mir07/mir07_data.html). Among these target genes, RhoE was identified, which is associated with regulation of the actin cytoskeleton and migration via alterations in cell motility (22). To validate whether RhoE is a direct target gene of miR-200b, a luciferase reporter vector containing the full length 3'-UTR of RhoE was cloned. miR-200b significantly suppressed the relative luciferase activity by targeting the 3'-UTR of RhoE. As shown in Fig. 2A, two predicted target sites of miR-200b have been identified, predictive target site 1 (from 1584 to 1591) and predictive target site 2 (from 1729 to 1735) in 3'UTR of RhoE. To analyze which predictive target site is regulated by miR-200b, a wild type RhoE 3'-UTR (containing the two prediction target sites) reporter construct and a mutated RhoE 3'-UTR reporter construct were generated. The results demonstrated that the first mutant reporter construct had less of an effect on luciferase activity, and the second mutant reporter construct had no effect on luciferase activity, as compared with the report vector containing the full 3'-UTR of RhoE. The luciferase assays revealed that miR-200b may directly target the 3'-UTR of RhoE by targeting predicted target sites 1 and 2 with both sites having the same effect. The effects of miR-200b on the endogenous expression of RhoE were subsequently examined by western blotting. Transfection of the HeLa cells with miR-200b mimics resulted in a marked decrease in the protein expression levels of RhoE (Fig. 2C). Furthermore, transfection with a miR-200b inhibitor upregulated the expression levels of RhoE in the cells. miRNA is known to post-transcriptionally regulate gene expression either via translational repression or mRNA 
A

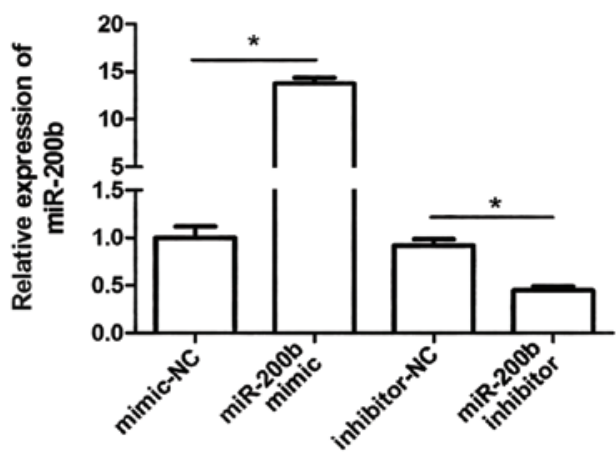

C

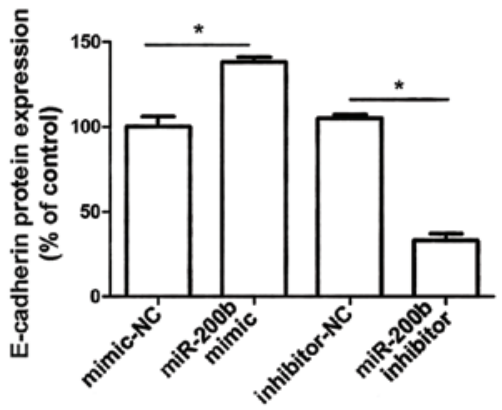

B

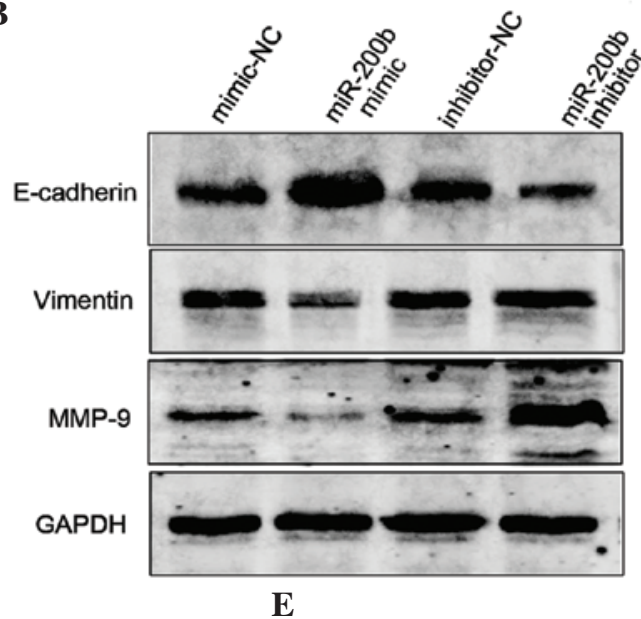

$\mathbf{F}$

D

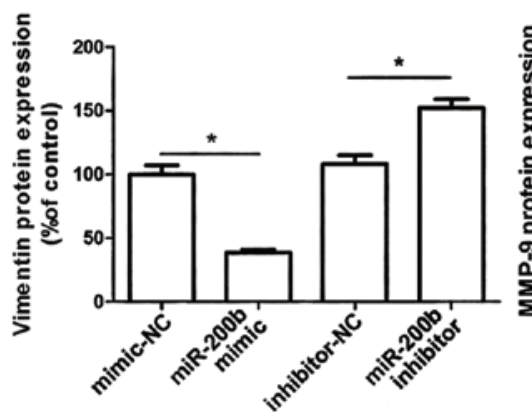

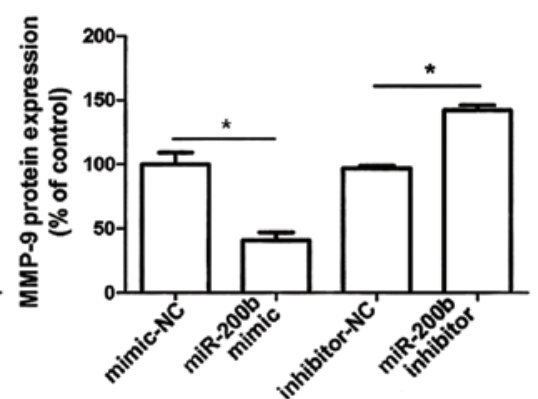
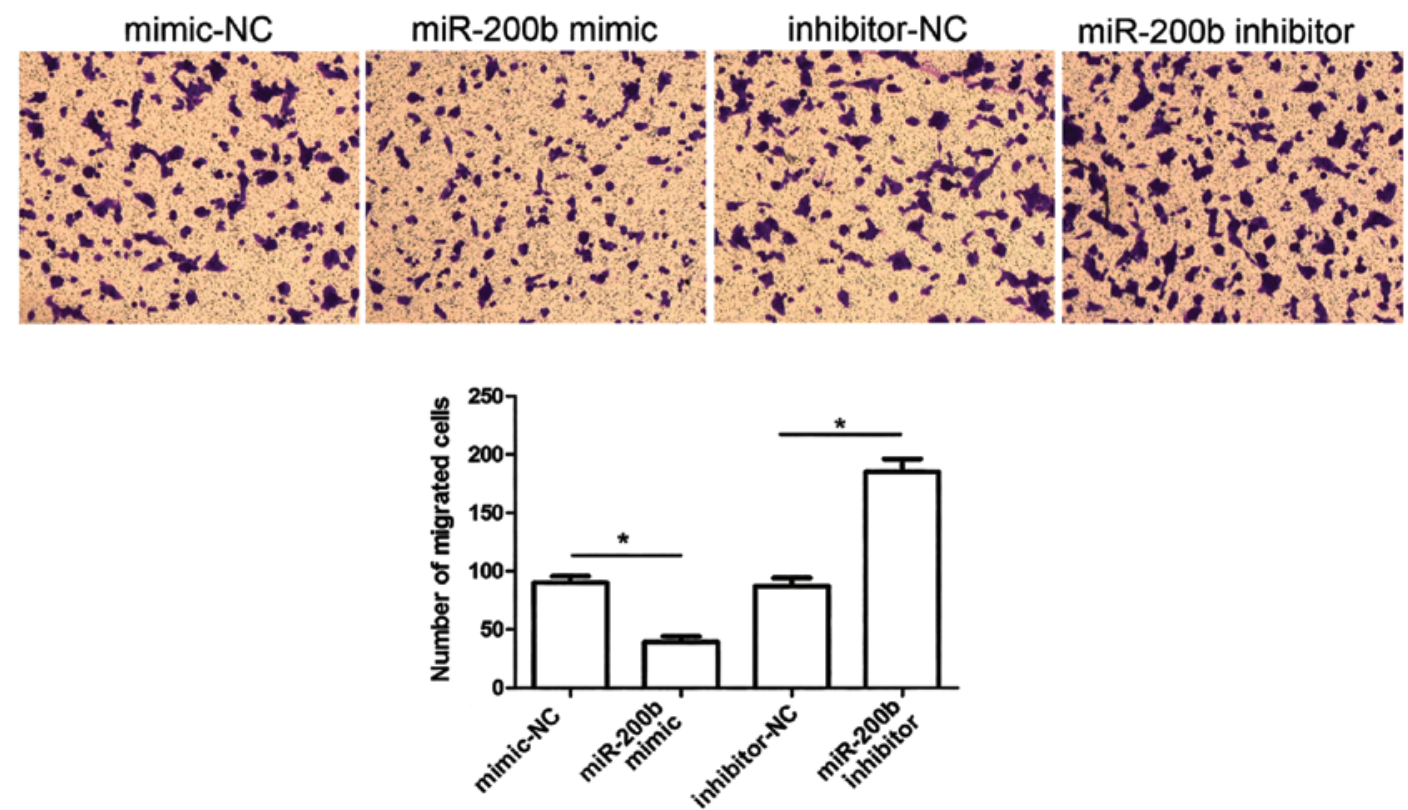

Figure 1. MicroRNA (miR)-200b expression mediated the epithelial-mesenchymal transition (EMT) of HeLa cells and regulated HeLa cell migration. (A) miR-200b expression levels following transient transfection with miR-200b mimics (100 nM) or inhibitors (200 nM), as determined by reverse transcription-quantitative polymerase chain reaction (RT-qPCR). (B) Transfection with miR-200b mimics or inhibitor alters protein expression levels, as determined by western blot analysis. RT-qPCR analysis of the expression levels of (C) E-cadherin, (D) vimentin and (E) matrix metalloproteinase (MMP)-9 post-transfection with miR-200b mimics, inhibitor, and a negative control (NC). (F) A Transwell migration system was used to evaluate migratory ability. Migration was decreased following the overexpression of miR-200b in HeLa cells. Images were captured using a phase contrast microscope under x 100 magnification. Data reflect the average number of cells that migrated in a representative experiment, measured in triplicate. Data are presented as the mean \pm standard error of the mean. ${ }^{*} \mathrm{P}<0.05$.

degradation (9). In the present study RhoE mRNA expression levels were decreased, as determined using RT-qPCR analysis (Fig. 2D). These results suggest that miR-200b could inhibit RhoE expression at the transcriptional level.
Silencing of RhoE inhibits EMT in HeLa cells. The present study aimed to investigate whether RhoE is the main factor affecting miR-200b-regulated cell migration in HeLa cells; therefore HeLa cells were transfected with siRNA-RhoE. As 
A

3'UTR of RhoE
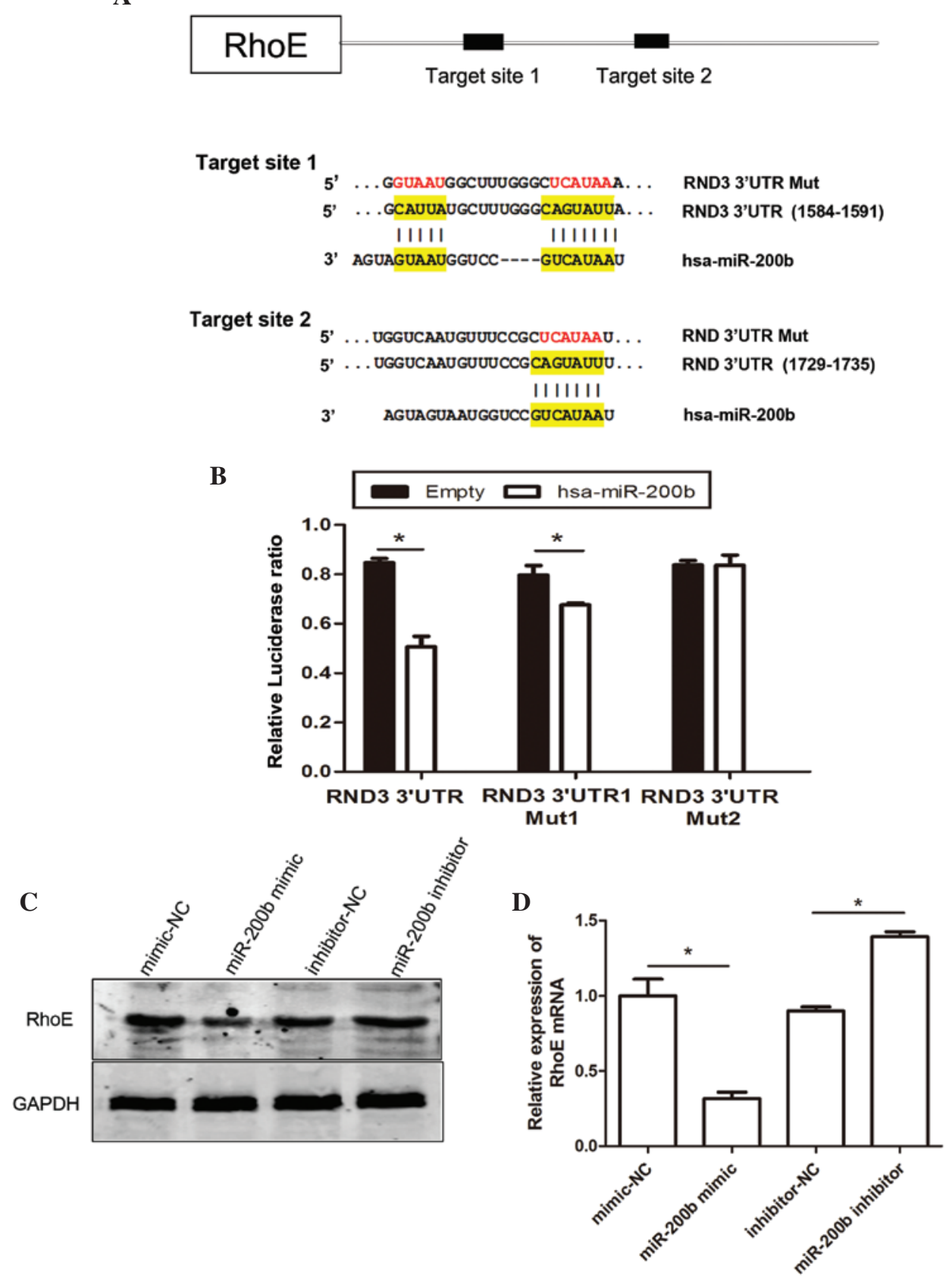

Figure 2. MicroRNA (miR)-200b directly targets RhoE. (A) Putative binding sites of miR-200b at the 1584-1591/1729-1735 position in the 3'-untranslated region (UTR) of RhoE were predicted by TargetScan (yellow). The mutated sites are shown in red. (B) Luciferase reporter assay indicated that miR-200b markedly suppressed luciferase activity by targeting both putative target sites of RhoE 3'-UTR. (C) Protein expression levels of RhoE, a known target of miR-200b, were significantly downregulated following transfection with miR-200b mimics. Transfection with an miR-200b inhibitor restored the protein expression of RhoE. (D) mRNA expression levels of RhoE post-transfection with miR-200b mimics, inhibitor, and a negative control (NC), as determined by reverse transcription-quantitative polymerase chain reaction. ${ }^{*} \mathrm{P}<0.05$.

shown in Fig. 3A, the expression levels of RhoE decreased by $>60 \%$ following transfection with siRNA-RhoE, as compared with the negative control-cells (Fig. 3A and B). In addition, siRNA-mediated RhoE downregulation resulted in increased E-cadherin expression and reduced vimentin protein expression in HeLa cells (Fig. 3C and D). Immunofluorescent labeling with an anti-E-cadherin antibody exhibited similar results; E-cadherin was significantly increased following transfection with siRNA-RhoE, as compared with the negative control HeLa cells (Fig. 3E). A Transwell assay was conducted to determine whether RhoE was able to influence the migratory ability of cervical cancer cells. The migratory ability of the Hela cells was markedly decreased when RhoE expression was silenced, as compared with the negative control group, thus indicating that downregulation of RhoE expression may suppress the cell migratory ability. These results suggest that downregulation of RhoE may inhibit EMT and cell migration in vitro.

miR-200b regulates EMT in HeLa cell by reducing the expression of RhoE. The present study aimed to investigate 
A

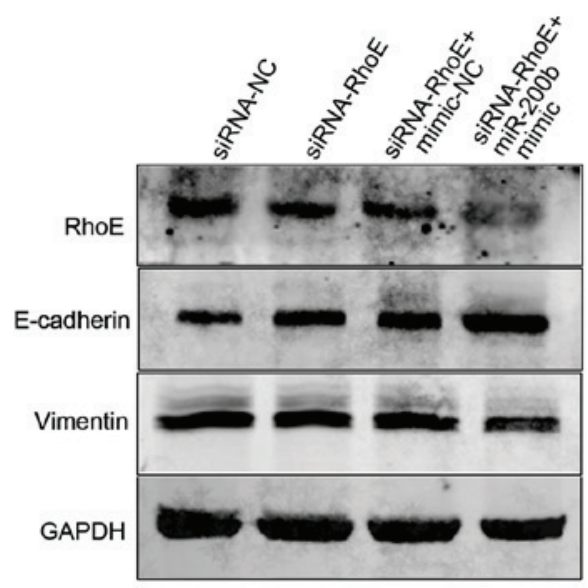

B

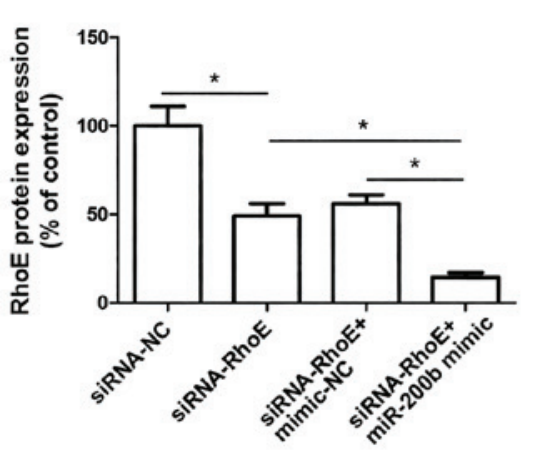

E

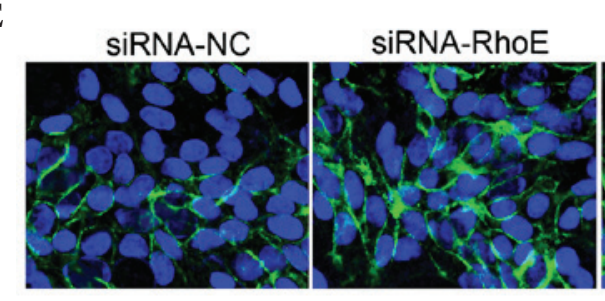

C

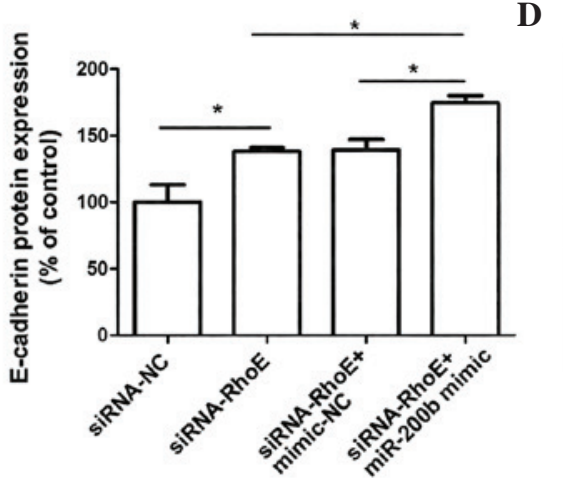

SiRNA-RhoE+ mimic-NC

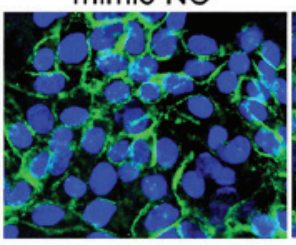

SiRNA-RhoE+ miR-200b mimic

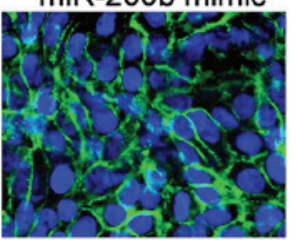

$\mathbf{F}$
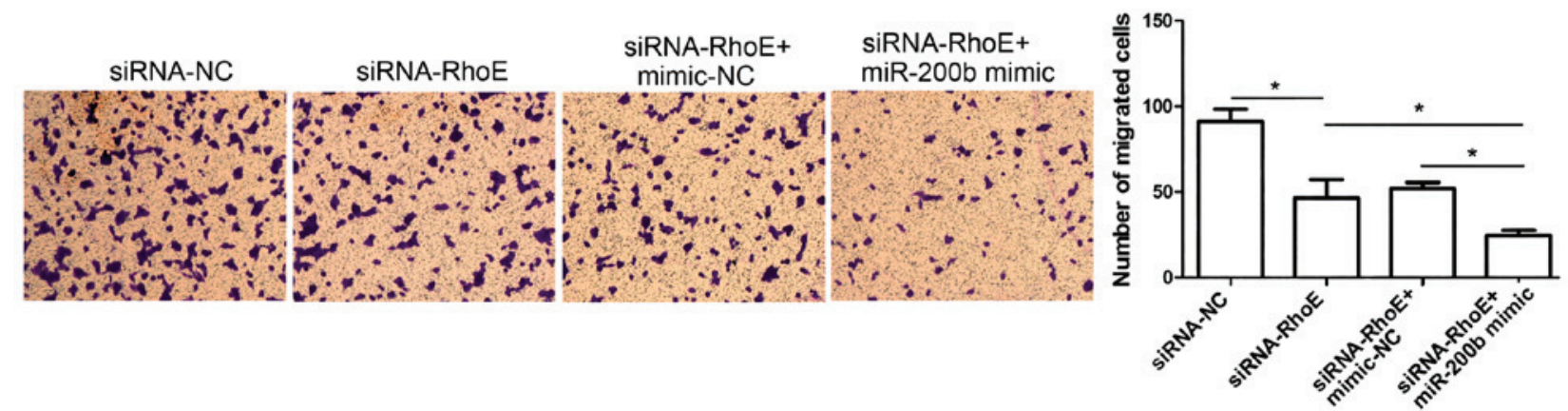

Figure 3. Knockdown of RhoE inhibits the epithelial-mesenchymal transition (EMT) of HeLa cells. MicroRNA (miR)-200b regulates the EMT of HeLa cells via altering the expression of RhoE. (A) Western blot analysis of RhoE, E-cadherin, vimentin and control glyceraldehyde 3-phosphate dehydrogenase (GAPDH) protein expression levels in HeLa cells following transfection with small interfering (si)RNA-RhoE. Total cell lysates were examined. Reverse transcription-quantitative polymerase chain reaction analysis of (B) RhoE, (C) E-cadherin and (D) vimentin expression following knockdown of RhoE by siRNA. (E) Immunofluorescent analysis for the detection of E-cadherin expression. Images were captured by phase contrast microscopy (magnification, $\mathrm{x}$ 200). (F) A Transwell migration assay was used to evaluate the migratory ability of the cells following various treatments. ${ }^{*}<0.05$. NC, negative control.

whether miR-200b influences the metastatic potential of HeLa cells via regulating the expression of RhoE. As presented in Fig. 3F, overexpression of miR-200b in siRNA-RhoE-transfected cells significantly impaired their migratory ability, as compared with the siRNA-RhoE cells transfected with mimic-NC. In addition, overexpression of miR-200b in the siRNA-RhoE-transfected cells resulted in an upregulation of E-cadherin expression, as compared with the mimic-NC 
group (Fig. 3A and F). These results indicate that miR-200b may function as a potent suppressor of migration in HeLa cells via regulation of RhoE expression.

\section{Discussion}

Metastasis is defined as a multistep biological process during which primary cancer cells form distant secondary tumors. Approximately $90 \%$ of patients with cancer succumb to metastatic disease (23); therefore, targeting metastasis is a key anticancer strategy. EMT, which is recognized as the initiation of cancer metastasis (24). Numerous studies have reported the role of miRNAs as promoters (25-27) and suppressors of metastasis (28-30); therefore, molecular regulation of miRNA expression may serve as a potential strategy for therapeutic intervention. High levels of miR-196a have been demonstrated to promote the migration and invasion of colorectal cancer cells via the repression of Hox genes (31). In addition, tumor suppressor miRNAs, including miR-29a, have also been reported, which inhibit cervical cell metastasis by targeting heat shock protein 47 (32). As a member of the miR-200 family, miR-200b has been identified as a powerful regulator of EMT in various types of cancer via the regulation of numerous genes; however, the function of miR-200b in cervical cancer remains to be determined. A significant correlation has previously been reported between the expression of miR-200 and the E-cadherin/vimentin ratio in NCI60 cells, and inhibition of endogenous miR-200 expression was sufficient to induce EMT $(14,15)$. Based on these findings, the present study hypothesized that miR-200b may be associated with the metastatic processes of cervical cancer.

To explore the mechanism underlying the effects of miR-200b on metastasis, potential miR-200b target genes were searched for using online miRNA target prediction databases, and numerous genes were identified. The present study selected one target gene of miR-200b, RhoE, which has a clear role in the regulation of the actin cytoskeleton and influences migration via alterations in cell motility, in order to determine the mechanisms underlying the effects of miR-200b on the regulation of EMT in cervical cancer cells. A previous study reported that RhoE may act as a target gene of miR-200b in regulating cell cycle progression of HeLa cells (33). In the present study, luciferase assays, RT-qPCR and western blotting provided direct evidence suggesting that miR-200b may bind to the 3'-UTR of RhoE, suppressing the expression of RhoE at the mRNA and protein levels. EMT is a key process that contributes to cancer metastasis, which is characterized by a loss of epithelial markers, including E-cadherin; an increase in mesenchymal markers, such as vimentin $(5,6)$; and an increase in migratory and invasive behavior. In the present study, overexpression of miR-200b in HeLa cells led to upregulated E-cadherin expression and downregulated vimentin expression. Conversely, transfection with an miR-200b inhibitor had the opposite effect on the HeLa cells. These findings suggested that miR-200b may be associated with the EMT and tumor metastasis of cervical cancer cells, and may serve as a potential therapeutic target for the treatment of invasive carcinoma.

A previous study has suggested that RhoE is implicated in cancer motility; however, the role of RhoE in cancer cell invasion remains at a preliminary stage (34). B-Raf-mediated upregulation of RhoE has been shown to promote melanoma cell invasion by reducing RhoA/RhoA kinase activity (35), whereas p53-mediated induction of RhoE has been linked to inhibition of cancer cell invasion (36), thus suggesting that RhoE may exert a dual function in modulating cell mobility and invasion. In colorectal cancer, RhoE expression has been shown to be significantly correlated with depth of invasion, and lymph node and distant metastasis (37), suggesting the role of RhoE in migration and invasion. Furthermore, RhoE expression was significantly reduced in colorectal cancer tissues, as compared with in normal tissues (38). The results of the present study demonstrated that siRNA-mediated knockdown of RhoE resulted in suppressive effects on EMT, as determined by reduced vimentin expression and increased E-cadherin expression. Furthermore, a Transwell migration assay indicated that knockdown of RhoE inhibited the cell migratory ability. These results indicated that miR-200b has a suppressive role in cell migration thereby influencing the EMT process, which may partly be due to direct inhibition of RhoE expression. The available data suggested that stress fibers act to maintain cell tension and mobility, and the loss of these actin structures directly leads to cell spreading (39). Previous studies have reported that loss of stress fibers is accompanied by rapid cell spreading $(40,41)$. It is well known that RhoA, which is a member of the Rho family, is involved in maintaining cell tension and a round morphology. A previous study demonstrated that RhoE may inhibit signaling downstream of RhoA to prevent RhoA from stimulating stress fiber formation, and time-lapse videomicroscopy has shown that following microinjection of RhoE, cells were much flatter and spread out, as compared with control cells (34). These findings suggested that RhoE may regulate cell spreading via the inhibition of RhoA signaling. Notably, overexpression of dominant-negative N-terminally truncated ROK $\alpha$, a downstream target for RhoA, induced cell spreading in HeLa and $3 T 3$ cells (41). However, further experiments are required.

In conclusion, the present study is the first, to the best of our knowledge, to describe the miR-200b/RhoE link and identify miR-200b as a regulator of EMT in cervical cancer cells by influencing the expression of RhoE. As a result, these findings may provide novel insights into the role of miR-200b in the development of cervical cancer and suggest that miR-200b may be considered an effective target for the treatment of patients with highly metastatic cervical cancer.

\section{Acknowledgements}

The authors are grateful for the support provided by the Natural Science Foundation of China (grant nos. 81302273 and 81201196), the Science and Technology Department of Hubei Province, China (grant no. ZRY039), the Health and Family Planning Commission of Hubei Province, China (grant no. 2012ZY02) and the Chinese Postdoctoral Science Foundation (grant no. 2011M500857).

\section{References}

1. American Cancer Society: Cancer Facts \& Figures 2015. Atlanta, American Cancer Society, 2015.

2. Siegel R, Naishadham D and Jemal A: Cancer statistics, 2013. CA Cancer J Clin 63: 11-30, 2013. 
3. Weitz J, Koch M, Debus J, Höhler T, Galle PR and Büchler MW: Colorectal cancer. Lancet 365: 153-165, 2005.

4. Fidler IJ: The organ microenvironment and cancer metastasis. Differentiation 70: 498-505, 2002.

5. Thiery JP: Epithelial-mesenchymal transitions in tumour progression. Nat Rev Cancer 2: 442-454, 2002.

6. Thiery JP, Acloque H, Huang RY and Nieto MA: Epithelial-mesenchymal transitions in development and disease. Cell 139: 871-890, 2009.

7. Hirohashi S: Inactivation of the E-cadherin-mediated cell adhesion system in human cancers. Am J Pathol 153: 333-339, 1998.

8. Hanahan D and Weinberg RA: Hallmarks of cancer: The next generation. Cell 144: 646-674, 2011.

9. Bartel DP: MicroRNAs: Target recognition and regulatory functions. Cell 136: 215-233, 2009.

10. Garzon R, Calin GA and Croce CM: MicroRNAs in cancer. Annu Rev Med 60: 167-179, 2009.

11. Esquela-Kerscher A and Slack FJ: Oncomirs - microRNAs with a role in cancer. Nat Rev Cancer 6: 259-269, 2006

12. Dykxhoorn DM: MicroRNAs and metastasis: Little RNAs go a long way. Cancer Res 70: 6401-6406, 2010.

13. Korpal M and Kang Y: The emerging role of miR-200 family of microRNAs in epithelial-mesenchymal transition and cancer metastasis. RNA Biol 5: 115-119, 2008.

14. Gravgaard KH, Lyng MB, Laenkholm AV, Søkilde R, Nielsen BS, Litman T and Ditzel HJ: The miRNA-200 family and miRNA-9 exhibit differential expression in primary versus corresponding metastatic tissue in breast cancer. Breast Cancer Res Treat 134 207-217, 2012.

15. Gregory PA, Bert AG, Paterson EL, Barry SC, Tsykin A, Farshid G, Vadas MA, Khew-Goodall Y and Goodall GJ: The miR-200 family and miR-205 regulate epithelial to mesenchymal transition by targeting ZEB1 and SIP1. Nat Cell Biol 10: 593-601, 2008.

16. Kong D, Li Y, Wang Z, Banerjee S, Ahmad A, Kim HR and Sarkar FH: miR-200 regulates PDGF-D-mediated epithelial-mesenchymal transition, adhesion, and invasion of prostate cancer cells. Stem Cells 27: 1712-1721, 2009.

17. Tryndyak VP, Beland FA and Pogribny IP: E-cadherin transcriptional down-regulation by epigenetic and microRNA-200 family alterations is related to mesenchymal and drug-resistant phenotypes in human breast cancer cells. Int J Cancer 126 2575-2583, 2010.

18. Hung CS, Liu HH, Liu JJ, Yeh CT, Chang TC, Wu CH, Ho YS, Wei PL and Chang YJ: MicroRNA-200a and -200b mediated hepatocellular carcinoma cell migration through the epithelial to mesenchymal transition markers. Ann Surg Oncol 20 (Suppl 3) S360-S368, 2013.

19. Kurashige J, Kamohara H, Watanabe M, Hiyoshi Y, Iwatsuki M, Tanaka Y, Kinoshita K, Saito S, Baba Y and Baba H: MicroRNA-200b regulates cell proliferation, invasion, and migration by directly targeting ZEB2 in gastric carcinoma. Ann Surg Oncol 19 (Suppl 3): S656-S664, 2012.

20. Adam L, Zhong M, Choi W, Qi W, Nicoloso M, Arora A, Calin G, Wang H, Siefker-Radtke A, McConkey D, et al: miR-200 expression regulates epithelial-to-mesenchymal transition in bladder cancer cells and reverses resistance to epidermal growth factor receptor therapy. Clin Cancer Res 15: 5060-5072, 2009.

21. Livak KJ and Schmittgen TD: Analysis of relative gene expression data using real-time quantitative PCR and the 2(-Delta Delta C(T)) Method. Methods 25: 402-408, 2001.

22. Chardin P: Function and regulation of Rnd proteins. Nat Rev Mol Cell Biol 7: 54-62, 2006.

23. Fidler IJ: Critical determinants of metastasis. Semin Cancer Biol 12: 89-96, 2002.
24. Arias AM: Epithelial mesenchymal interactions in cancer and development. Cell 105: 425-431, 2001

25. Ma L, Teruya-Feldstein J and Weinberg RA: Tumour invasion and metastasis initiated by microRNA-10b in breast cancer. Nature 449: 682-688, 2007.

26. Huang Q, Gumireddy K, Schrier M, Ie Sage C, Nagel R, Nair S, Egan DA, Li A, Huang G, Klein-Szanto AJ, et al: The microRNAs $\mathrm{miR}-373$ and miR-520 c promote tumour invasion and metastasis. Nat Cell Biol 10: 202-210, 2008.

27. Gaziel-Sovran A, Segura MF, Di Micco R, Collins MK, Hanniford D, Vega-Saenz de Miera E, Rakus JF, Dankert JF, Shang S, Kerbel RS, et al: miR-30b/30d regulation of GalNAc transferases enhances invasion and immunosuppression during metastasis. Cancer Cell 20: 104-118, 2011.

28. Li N, Fu H, Tie Y, Hu Z, Kong W, Wu Y and Zheng X: miR-34a inhibits migration and invasion by down-regulation of c-Met expression in human hepatocellular carcinoma cells. Cancer Lett 275: 44-53, 2009.

29. Fang JH, Zhou HC, Zeng C, Yang J, Liu Y, Huang X, Zhang JP, Guan XY and Zhuang SM: MicroRNA-29b suppresses tumor angiogenesis, invasion, and metastasis by regulating matrix metalloproteinase 2 expression. Hepatology 54: 1729-1740, 2011.

30. Tavazoie SF, Alarcón C, Oskarsson T, Padua D, Wang Q, Bos PD, Gerald WL and Massagué J: Endogenous human microRNAs that suppress breast cancer metastasis. Nature 451: $147-152,2008$

31. Schimanski CC, Frerichs K, Rahman F, Berger M, Lang H, Galle PR, Moehler M and Gockel I: High miR-196a levels promote the oncogenic phenotype of colorectal cancer cells. World J Gastroenterol 15: 2089-2096, 2009.

32. Yamamoto N, Kinoshita T, Nohata N, Yoshino H, Itesako T, Fujimura L, Mitsuhashi A, Usui H, Enokida H, Nakagawa M, et al: Tumor-suppressive microRNA-29a inhibits cancer cell migration and invasion via targeting HSP47 in cervical squamous cell carcinoma. Int J Oncol 43: 1855-1863, 2013.

33. Xia W, Li J, Chen L, Huang B, Li S, Yang G, Ding H, Wang F, Liu N, Zhao Q, et al: MicroRNA-200b regulates cyclin D1 expression and promotes S-phase entry by targeting RND3 in HeLa cells. Mol Cell Biochem 344: 261-266, 2010.

34. Guasch RM, Scambler P, Jones GE and Ridley AJ: RhoE regulates actin cytoskeleton organization and cell migration. Mol Cell Biol 18: 4761-4771, 1998.

35. Klein RM and Aplin AE: Rnd3 regulation of the actin cytoskeleton promotes melanoma migration and invasive outgrowth in three dimensions. Cancer Res 69: 2224-2233, 2009.

36. Gadea G, de Toledo M, Anguille C and Roux P: Loss of p53 promotes RhoA-ROCK-dependent cell migration and invasion in 3D matrices. J Cell Biol 178: 23-30, 2007.

37. Zhou J, Yang J, Li K, Mo P, Feng B, Wang X, Nie Y and Fan D: RhoE is associated with relapse and prognosis of patients with colorectal cancer. Ann Surg Oncol 20: 175-182, 2013.

38. Luo H, Zou J, Dong Z, Zeng Q, Wu D and Liu L: Up-regulated miR-17 promotes cell proliferation, tumour growth and cell cycle progression by targeting the RND3 tumour suppressor gene in colorectal carcinoma. Biochem J 442: 311-321, 2012.

39. Katoh H, Harada A, Mori K and Negishi M: Socius is a novel Rnd GTPase-interacting protein involved in disassembly of actin stress fibers. Mol Cell Biol 22: 2952-2964, 2002

40. Leung T, Chen XQ, Manser E and Lim L: The p160 RhoA-binding kinase ROK alpha is a member of a kinase family and is involved in the reorganization of the cytoskeleton. Mol Cell Biol 16: 5313-5327, 1996.

41. Ridley AJ and Hall A: The small GTP-binding protein rho regulates the assembly of focal adhesions and actin stress fibers in response to growth factors. Cell 70: 389-399, 1992. 